\title{
Lipocalin-2 is associated with radioresistance in oral cancer and lung cancer cells
}

\author{
MASASHI SHIIBA $^{1,2}$, KENGO SAITO $^{3}$, KAZUAKI FUSHIMI ${ }^{1}$, TAKASHI ISHIGAMI ${ }^{1}$, KEIJI SHINOZUKA ${ }^{1}$, \\ DAI NAKASHIMA ${ }^{1}$, YUKINAO KOUZU ${ }^{1}$, HIROFUMI KOIKE ${ }^{1}$, ATSUSHI KASAMATSU ${ }^{1}$, YOSUKE SAKAMOTO ${ }^{1}$, \\ KATSUNORI OGAWARA $^{1}$, KATSUHIRO UZAWA ${ }^{1}$, YUICHI TAKIGUCHI ${ }^{2}$ and HIDEKI TANZAWA ${ }^{1}$ \\ Departments of ${ }^{1}$ Clinical Molecular Biology, ${ }^{2}$ Medical Oncology and ${ }^{3}$ Molecular Virology, \\ Graduate School of Medicine, Chiba University, Chuo-ku, Chiba 260-8670, Japan
}

Received November 12, 2012; Accepted December 21, 2012

DOI: 10.3892/ijo.2013.1815

\begin{abstract}
The aim of the present study was to identify a target molecule that could predict the efficacy of radiotherapy in oral squamous cell carcinoma (OSCC). We used DNA microarray analysis to identify differences in gene expression after X-ray irradiation. We compared the gene expression profiles between X-ray (8 Gy)-irradiated Ca9-22 cells (an OSCC-derived cell line) and unirradiated Ca9-22 cells. A total of 167 genes with a 2-fold higher level of expression induced by X-ray irradiation were identified. Lipocalin-2 (LCN2) had the greatest increase in expression after X-ray irradiation, and it was categorized in a network that has cancer-related functions with the Ingenuity Pathway Analysis tool. Upregulated expression of LCN2 mRNA was validated by real-time quantitative reverse transcriptasepolymerase chain reaction (qRT-PCR) analysis. When the LCN2 gene was knocked down in OSCC cells (Ca9-22 and HSC-2) and lung cancer cells (A549) by using small interfering RNA, the radiosensitivity of these cells was enhanced. Our findings suggest that the overexpression of LCN2 is likely associated with radioresistance in oral cancer and lung cancer cells, and that LCN2 expression levels could be used to predict radioresistance. Thus, regulating the expression or function of LCN2 could enhance the radiation response, resulting in a favorable outcome of radiotherapy.
\end{abstract}

\section{Introduction}

Although radiotherapy for oral squamous cell carcinoma (OSCC) is effective in certain patients, some patients do not respond to radiotherapy. The discrepancy between responders and non-responders mostly depends on the radiosensitivity of tumor cells. Thus, it is crucial to determine the mechanism of radiosensitivity and to identify molecules that regulate radiotherapy

Correspondence to: Dr Masashi Shiiba, Department of Clinical Molecular Biology, Graduate School of Medicine, Chiba University, 1-8-1 Inohana, Chuo-ku, Chiba 260-8670, Japan

E-mail: m.shiiba@faculty.chiba-u.jp

Key words: lipocalin-2, oral squamous cell carcinoma responsiveness. Many studies have reported a correlation between gene expression and the response to radiotherapy (1-3). The products of these effector genes participate in a radiation-induced response (3-5), which includes necrosis, apoptosis, cell cycle arrest and DNA repair (5-7). In oral malignancies including OSCCs, cyclooxygenase-2 (COX-2) (8), surviving (9), DNA contact mutation of p53 (10), tumor suppressor homologue p63 (11), tongue cancer resistance-associated protein 1 (TCRP1) (12) and intercellular adhesion molecule 2 (ICAM2) (13) may be associated with radioresistance.

Lipocalins are a functionally diverse family of proteins that can bind to surface receptors and a variety of lipophilic substances. Lipocalins are upregulated in a number of pathological conditions and may function as transporters of essential factors (14) and regulators of cell homoeostasis and the modulation of the immune response (15). Lipocalin-2 (LCN2, also known as neutrophil gelatinase-associated lipocalin: NGAL), a member of the lipocalin family, exists as a $25-\mathrm{kDa}$ monomer, a 46-kDa disulphide-linked homodimer and a 135-kDa disulphide-linked heterodimer with neutrophil gelatinase-B (16). LCN2 is thought to be an acute phase protein (17), the expression of which is upregulated in epithelial cells under diverse inflammatory conditions including appendicitis, inflammatory bowel disease and diverticulitis (18). Lipocalins affect cellular proliferation and differentiation, and may be involved in the development of carcinomas (19). Previous studies have reported that LCN2 is expressed in human colorectal cancer (18), pancreatic cancer cells, colorectal and hepatic tumors (20), and human ovarian cancer cell lines (21). In head and neck tumors, Hiromoto et al reported that LCN2 expression was strongly upregulated in well-differentiated OSCC tissues and moderately to weakly upregulated in moderately to poorly differentiated OSCC tissues, while its expression was weak or very weak in normal mucosa and leukoplakia (22). It was recently reported that the upregulation of LCN2 expression in human adenocarcinoma A549 cells was accompanied by apoptosis induced by several reagents and that the induction of LCN2 represents a survival response (14).

In the current study, we performed DNA microarray analysis to assess gene expression changes in OSCC cells after X-ray irradiation. The genes identified were subjected to network and gene ontology analysis, and functional analyses 
were performed to clarify whether the candidate molecule is related to radioresistance by gene silencing.

\section{Materials and methods}

Cell lines and culture conditions. The human OSCC-derived cell lines Ca9-22, HSC-2 and the human lung cancer cells A549, were prepared for this study (Human Science Research Resources Bank, Osaka, Japan). The cells were maintained in Dulbecco's modified Eagle's medium F-12 HAM (Sigma Chemical Co., St. Louis, MO, USA) and supplemented with $10 \%$ heat-inactivated fetal bovine serum and $50 \mathrm{U} / \mathrm{ml}$ penicillin and streptomycin. All cultures were grown at $37^{\circ} \mathrm{C}$ in a humidified atmosphere of $5 \% \mathrm{CO}_{2}$.

$X$-ray irradiation. The cells were irradiated using X-ray irradiation equipment (MBR-1520R-3, Hitachi, Tokyo, Japan) operated at $150 \mathrm{~V}$ and $20 \mathrm{~mA}$ with AL filtration at a dose of $2.1 \mathrm{~Gy} / \mathrm{min}$.

Isolation of RNA. Total RNA was extracted with TRIzol reagent (Invitrogen Life Technologies, Carlsbad, CA, USA) from irradiated and unirradiated cells $4 \mathrm{~h}$ after irradiation, according to the manufacturer's instructions. The quality of total RNA was determined by Bioanalyzer (Agilent Technologies, Palo Alto, CA, USA).

Preparation of $c D N A$. Total RNA was extracted using TRIzol reagent. Five micrograms of total RNA from each sample was reverse transcribed to cDNA using Ready-to-Go You-Prime first-strand beads (GE Healthcare, Buckinghamshire, UK) and oligo (dT) primer (Sigma Genosys, Ishikari, Japan), according to the manufacturer's protocol.

Hybridization of RNAs to oligonucleotide arrays. For microarray analysis, $4 \mathrm{~h}$ after irradiation ( $8 \mathrm{~Gy}$ ) was selected as the time-point at which to monitor the early response of $\mathrm{Ca} 9-22$ cells to X-ray irradiation and to identify differentially expressed early genes that mediate cellular events such as DNA repair and apoptosis. We used Human Genome U133A Array GeneChip oligonucleotide arrays (Affymetrix, Santa Clara, CA, USA). This GeneChip, containing 22,283 probe sets, analyzes the expression level of over 18,400 transcripts and variants, including 14,500 well-characterized human genes. For hybridization, $20 \mu \mathrm{g}$ of total RNA per sample was prepared according to the manufacturer's protocol (Affymetrix). Fragmented cRNA (15 $\mu \mathrm{g}$ of each) was hybridized to the Human Genome oligonucleotide arrays. The arrays were stained with phycoerythrin-streptavidin and the signal intensity was amplified by treatment with a biotinconjugated anti-streptavidin antibody, followed by a second staining using phycoerythrin-streptavidin. The arrays stained a second time were scanned using the Affymetrix GeneChip Scanner 3000.

Analysis of microarray data. GeneChip analysis was performed based on the Affymetrix GeneChip Manual with Microarray Analysis Suite 5.0, Data Mining Tool 2.0 and Microarray Database software. The genes on the GeneChip were globally normalized and scaled to a signal intensity of 500. The Microarray Analysis Suite software used Wilcoxon's test to generate detected (present or absent) calls and used the calls to statistically determine if a transcript was expressed or not. After being filtered through a 'present' call $(\mathrm{P}<0.05)$, the expression data were analyzed using GeneChip Operating Software 1.1 (Affymetrix) and GeneSpring 6.1 (Silicon Genetics, Redwood City, CA, USA). Fold changes were calculated by comparing transcripts between irradiated Ca9-22 cells and unirradiated Ca9-22 cells. We identified 167 genes differentially expressed 2.0 -fold or more by X-ray irradiation. The genes, which were identified by microarray analyses, were analyzed for network and gene ontology by Ingenuity Pathway Analysis (IPA) software (Ingenuity Systems, Mountain View, CA, USA) to identify networks of interacting genes. Gene accession numbers were imported into the IPA software. The genes were categorized based on location, cellular components, and reported or suggested biochemical, biologic and molecular functions using the software. The identified genes were mapped to the genetic networks available in the IPA database and then ranked by score. The score is the probability that a collection of genes equal to or greater than the number in a network could be achieved by chance alone. A score of 3 indicates that there is a 1/1,000 chance that the focus genes are in a network due to random chance. Therefore, scores of 3 or higher have a $99.9 \%$ confidence level of not being generated by random chance alone. This score was used as the cut-off for identifying gene networks.

Analysis of $m R N A$ expression by real-time quantitative reverse transcriptase-polymerase chain reaction ( $q R T-P C R)$. Real-time qRT-PCR was performed to validate mRNA expression with a single method using a LightCycler FastStart DNA Master SYBR-Green 1 kit (Roche Diagnostics GmbH, Mannheim, Germany), according to the procedure provided by the manufacturer. Oligonucleotides used as primers were 5'-GCTGACTTC GGAACTAAAGGAGAA-3' and 5'-GGGAAGACGATGTG GTTTTCA-3' for LCN2 mRNA and 5'-CATCTCTGCCCCC TCTGCTGA-3' and 5'-GGATGACCTTGCCCACAGCCT-3' for glyceraldehyde-3-phosphate dehydrogenase (GAPDH) mRNA. Using LightCycler (Roche Diagnostics $\mathrm{GmbH}$ ) apparatus, the experiment was carried out in a final volume of $20 \mu \mathrm{l}$ of reaction mixture consisting of $2 \mu \mathrm{l}$ of FirstStart DNA Master SYBR-Green I mix, $3 \mathrm{mM} \mathrm{MgCl}$ and $1 \mu \mathrm{l}$ of the primers, according to the manufacturer's instructions. Subsequently, the reaction mixture was loaded into glass capillary tubes and subjected to an initial denaturation at $95^{\circ} \mathrm{C}$ for $10 \mathrm{~min}$, followed by 45 rounds of amplification at $95^{\circ} \mathrm{C}(10 \mathrm{sec})$ for denaturation, 62 to $64^{\circ} \mathrm{C}(10 \mathrm{sec})$ for annealing, and $72^{\circ} \mathrm{C}$ for extension. The transcript amount for the genes differentially expressed in the microarray analysis was estimated from the respective standard curves and normalized to the GAPDH transcript amount determined in corresponding samples.

Transfection of siRNAs in cells. SMARTpool siRNA targeting $L C N 2$ consists of four siRNAs targeting multiple sites on $L C N 2$ ( $L C N 2$-siRNAs). The sequences for $L C N 2$-siRNAs are 5 '-UGG GCAACAUUAAGAGUUAUU-3' (sense), 5'-PUAACUCUU AAUGUUGCCCAUU-3' (antisense), 5'-GAGCUGACUUC GGAACUAAUU-3' (sense), 5'-PUUAGUUCCGAAGUCAGC UCUU-3' (antisense), 5'-GAAGACAAAGACCCGCAAAUU-3' (sense), 5'-PUUUGCGGGUCUUUGUCUUCUU-3' (antisense), 5'-GAAGACAAGAGCUACAAUGUU-3' (sense) and 5'-PCAU 
UGUAGCUCUUGUCUUCUU-3' (antisense) (On-Target plus SMARTpool, L-003679-00-0005, Human LCN2, NM005564). Positive and negative control siRNAs were purchased (Dharmacon, Lafayette, CO, USA). Two negative controls were used, vehicle control and siControl non-targeting siRNA pool (D-001206-13-20; siNT). Cyclophilin B (siControl cyclophilin B, siCyclo) was used as a positive silencing control to ascertain the transfection efficiency in each experiment. Cells were transfected with siRNAs using DharmaFECTl reagent (Dharmacon). Cells were plated in antibiotic-free Dulbecco's modified Eagle's medium F-12 HAM at a density of 200,000 cells $/ 4 \mathrm{ml}$ in $60-\mathrm{mm}$ dishes. After $24 \mathrm{~h}$, the cells were transfected with $100 \mathrm{nmol} / \mathrm{l}$ siRNA in DharmaFECTl reagent, according to the manufacturer's instructions. Briefly, $8 \mu \mathrm{l}$ DharmaFECTl was diluted in $392 \mu 1$ of serum-free medium and incubated at room temperature for $5 \mathrm{~min}$. In a separate tube, $200 \mu \mathrm{l}$ of $2 \mu \mathrm{mol} / 1 \mathrm{siRNA}$ was diluted in $200 \mu \mathrm{l}$ of serum-free medium at room temperature for $5 \mathrm{~min}$. Diluted DharmaFECT1 ( $400 \mu \mathrm{l})$ was added to the diluted siRNA and the complex was incubated for $20 \mathrm{~min}$ at room temperature. The cells were washed with antibiotic-free Dulbecco's modified Eagle's medium F-12 HAM and $3.2 \mathrm{ml}$ antibiotic-free Dulbecco's modified Eagle's medium F-12 HAM was added to each dish. siRNA + DharmaFECT1 complex $(800 \mu \mathrm{l})$ was added gently to the dish. The final concentration of siRNA was $100 \mathrm{nmol} / \mathrm{l}$. Control cells were treated with medium only, the $100 \mathrm{nmol} / \mathrm{l}$ non-targeted siRNA (siNT negative control), and the $100 \mathrm{nmol} / \mathrm{l}$ cyclophilin B siRNA (positive silencing control). After $4 \mathrm{~h}$ of transfection, the medium of cells treated with $L C N 2$-siRNA (siLCN2) and control cells was replaced with fresh medium, and cells were incubated at $37^{\circ} \mathrm{C}$ in $5 \% \mathrm{CO}_{2}$ for $120 \mathrm{~h}$ before the experiments.

Western blot analysis. Cells were lysed in buffer [10 $\mathrm{mM}$ Tris base (pH 8.0), $400 \mathrm{mM} \mathrm{NaCl}, 3 \mathrm{mM} \mathrm{MgCl} 2,0.5 \%$ Nonidet P-40 (Sigma), $100 \mathrm{mM}$ phenylmethylsulfonyl fluoride and $0.01 \%$ protease inhibitor cocktail (Sigma)] at $4^{\circ} \mathrm{C}$ for $10 \mathrm{~min}$. Protein extracts were electrophoresed on $11 \%$ sodium dodecyl sulfate-polyacrylamide gel electrophoresis gels and transferred to polyvinylidene fluoride (PVDF) membranes (Bio-Rad, Hercules, CA, USA). Immunoblot PVDF membranes were washed with $0.1 \%$ Tween-20 in TBS, and affinity-purified mouse anti-human LCN2 monoclonal antibody (Santa Cruz Biotechnology, Santa Cruz, CA, USA) was added at 1:100 and incubated overnight at room temperature. PVDF membranes were washed again and incubated with a 1:5,000 of horseradish peroxidase-conjugated anti-mouse IgG Envision+ (Dako Japan Inc., Kyoto, Japan) as a secondary antibody for $2 \mathrm{~h}$ at room temperature. Finally, membranes were incubated with enhanced chemiluminescence (ECL)+ horseradish peroxidase substrate solution included in the ECL+ kit (GE Healthcare) and immunoblotting was visualized by exposing the membrane to Hyperfilm (GE Healthcare).

Cell survival assay. Cells were transfected as described previously with the vehicle, siNT and siLCN2. At $96 \mathrm{~h}$ after transfection, the cells were trypsinized, counted and the appropriate number of cells was plated in $60-\mathrm{mm}$ dishes and allowed to attach for $24 \mathrm{~h}$. After $24 \mathrm{~h}$, the cells were irradiated $(2,4,6,8 \mathrm{~Gy})$ and incubated for 8 to 10 days. The colonies were stained with crystal violet (Sigma Chemical Co.) and colonies of 50 cells or greater were counted. Clonogenic fractions of irradiated cells were normalized to the plating efficiency of unirradiated controls.

Cellular proliferation assay. To determine the effect of siLCN2 transfection on cell proliferation, Ca9-22 cells transfected with non-targeting or LCN2 siRNA were seeded in 12-well plates at a density of $1 \times 10^{4}$ viable cells per well. At the indicated time point, cells were trypsinized and counted using a hemocytometer in triplicate samples.

\section{Results}

DNA microarray and network analysis. The gene expression profile in the irradiated OSCC-derived cell line Ca9-22, was analyzed with DNA microarray analysis. A total of 167 genes were overexpressed after X-ray irradiation ( $8 \mathrm{~Gy}$ ) by at least 2-fold when compared with unirradiated Ca9-22 cells, while 14 genes were upregulated more than 5 -fold (Table I).

We then investigated whether the 167 genes that were overexpressed by at least 2-fold interacted biologically by performing genetic network analysis with the IPA tool. Among these genes, 82 genes were mapped to six genetic networks in which functional relationships between gene products have been reported (Fig. 1). The six networks were highly significant and contained some common biological functions, such as cancer, cell death, cellular growth and proliferation (Table II). $L C N 2$, which was mapped to network 2 , had the greatest increase in expression after X-ray irradiation (Table I).

Validation of microarray data by real-time qRT-PCR analysis. To verify the gene expression identified in the DNA microarray analysis, real-time qRT-PCR was performed by using the same RNA that was used in the DNA microarray analysis. Consistent with the results of DNA microarray analysis, there was a significant increase in the expression levels of $L C N 2$ in X-ray irradiated Ca9-22 cells as compared with unirradiated Ca9-22 cells (Fig. 2). The data are expressed as the mean \pm standard deviation (SD) of two independent experiments with samples in triplicate.

Functional analysis in siLCN2-tranfected cells. To determine whether LCN2 silencing contributes to radiation sensitivity, cells were transfected with siRNAs and screened for their ability to downregulate target protein expression. To ascertain that RNA inhibition conditions were optimal and transfection efficiency was satisfactory, cyclophilin B siRNA was used as a positive control in each experiment. In Ca9-22, HSC-2 and A549 cells transfected with cyclophilin B siRNA (siCyclo), the cyclophilin B protein level was reduced significantly as compared to the vehicle or siNT controls (siNT) (Fig. 3). LCN2 protein expression was examined by western blot analysis in Ca9-22, HSC-2 and A549 cells $120 \mathrm{~h}$ after transfection with siRNAs (Figs. 4-6). LCN2 protein levels in vehicle and siNT-transfected cells were comparable to that of LCN2 in non-treated cells. In addition, in cells transfected with $100 \mathrm{nmol} / 1 \mathrm{siLCN} 2$, the LCN2 protein level was reduced significantly as compared with the positive and negative control cells. These transfected cells were subjected to functional analysis to reveal the effect of LCN2 gene silencing in radiation response. Survival of Ca9-22, HSC-2 and A549 cells transfected with siLCN2 at $120 \mathrm{~h}$ decreased significantly $(\mathrm{P}<0.01$, Student's 
Table I. Differentially expressed genes (fold change $>5$ ).

\begin{tabular}{|c|c|c|c|}
\hline $\begin{array}{l}\text { Affymetrix } \\
\text { no. }\end{array}$ & Symbol & Name & $\begin{array}{c}\text { Fold } \\
\text { change }^{\mathrm{a}}\end{array}$ \\
\hline 212531_at & $L C N 2$ & Lipocalin 2 (oncogene $24 \mathrm{p} 3$ ) & 14.2 \\
\hline 204580_at & MMP12 & Matrix metalloproteinase 12 (macrophage elastase) & 12.2 \\
\hline 220026_at & CLCA4 & Chloride channel, calcium activated, family member 4 & 11.6 \\
\hline 220523_at & FLJ 22843 & Hypothetical protein FLJ22843 & 8.4 \\
\hline 211708_s_at & CESK1 & T-complex protein 1 & 8.2 \\
\hline 211708_s_at & C13orf10 & Chromosome 13 open reading frame 10 & 7.0 \\
\hline 216697_at & TRIO & Triple functional domain (PTPRF interacting) & 6.7 \\
\hline 200831_s_at & $S C D$ & Stearoyl-CoA desaturase ( $\Delta^{9}$ desaturase) & 6.6 \\
\hline 214605_x_at & GPRl & G protein-coupled receptor 1 & 6.5 \\
\hline 202815_s_at & HIS1 & HMBA-inducible & 6.1 \\
\hline 213112_s_at & SQSTM1 & Sequestosome 1 & 5.9 \\
\hline 202828_s_at & MMP14 & Matrix metalloproteinase 14 (membrane-inserted) & 5.7 \\
\hline 202820_at & $A H R$ & Aryl hydrocarbon receptor & 5.7 \\
\hline 205660_at & OASL & 2'-5'-oligoadenylate synthetase-like & 5.0 \\
\hline
\end{tabular}

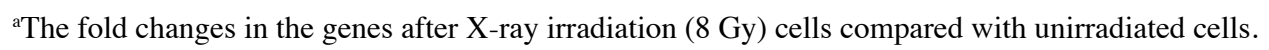
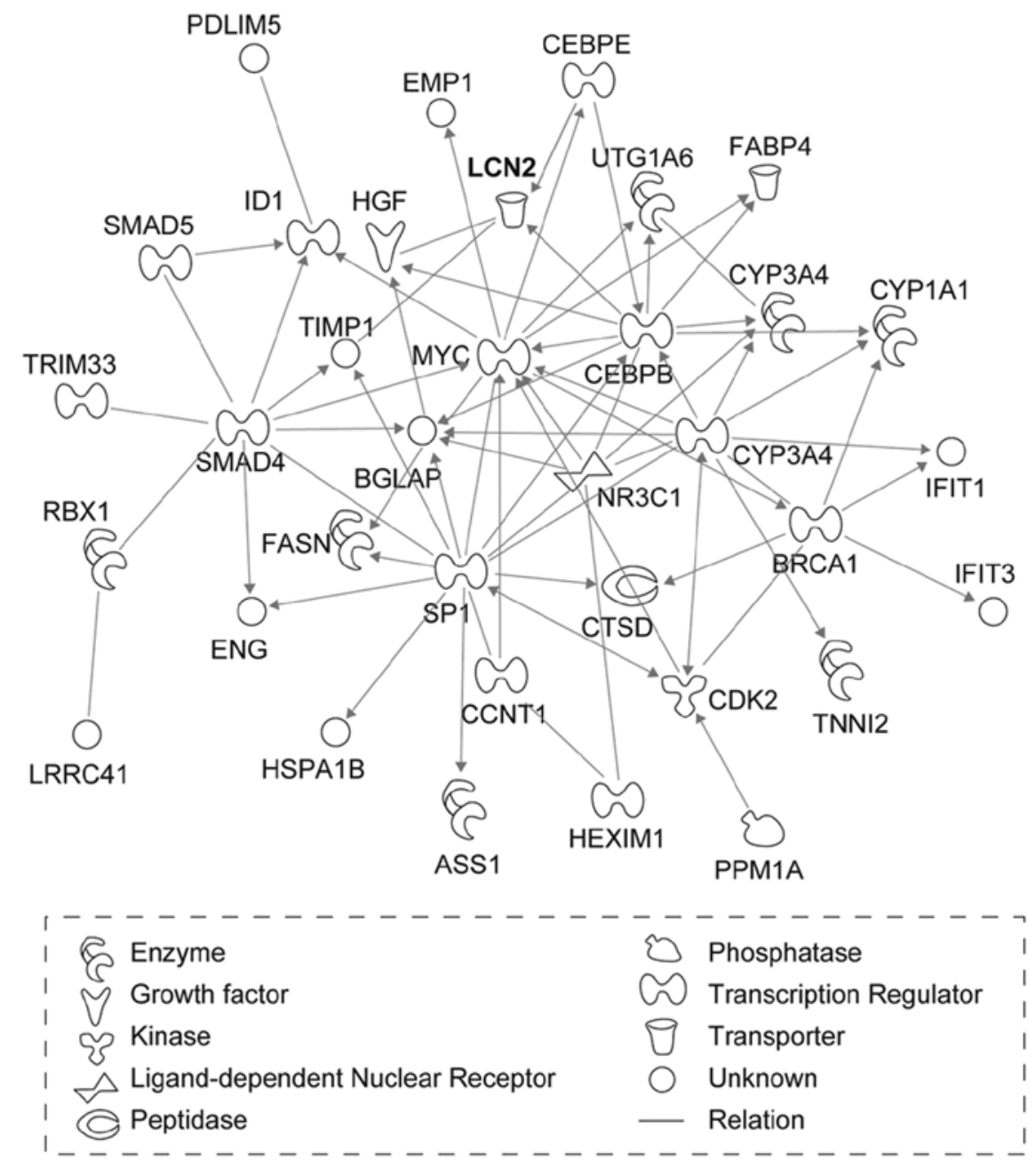

Figure 1. The network of molecules (including LCN2) categorized by IPA software. The identified network shows the classification of molecules and their interactions. 
Table II. Genetic networks in the X-ray irradiated oral squamous cell carcinoma cells.

\begin{tabular}{|c|c|c|c|}
\hline Network & Gene & Function & \\
\hline 1 & 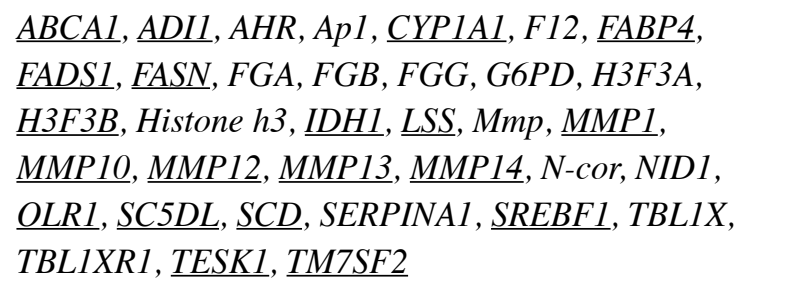 & $\begin{array}{l}\text { Post-translational modification, } \\
\text { genetic disorder, } \\
\text { hematological disease }\end{array}$ & 40 \\
\hline 2 & $\begin{array}{l}\underline{A S S 1}, B G L A P, B R C A 1, C C N T 1, C D K 2, C E B P B, \\
C E B P E, \underline{C T S D}, \underline{C Y P 1 A 1}, C Y P 3 A 4, \underline{E M P 1}, E N G, \\
\underline{F A B P 4}, \underline{F A S N}, \underline{H E X I M 1}, H G F, \underline{H S P A 1 B}, I D 1, \underline{I F I T 1}, \\
\underline{I F I T 3}, \underline{L C N 2}, \underline{\text { LRRC41 }}, M Y C, N R 3 C 1, \underline{P D L I M 5}, \\
\underline{P P M 1 A}, R B X 1, S M A D 4, S M A D 5, S M A R C A 4, \\
S P 1, T I M P 1, \underline{T N N I 2}, \underline{T R I M 33}, \underline{U G T 1 A 6}\end{array}$ & $\begin{array}{l}\text { Cancer, gene expression, } \\
\text { cellular growth and } \\
\text { proliferation }\end{array}$ & 30 \\
\hline 3 & 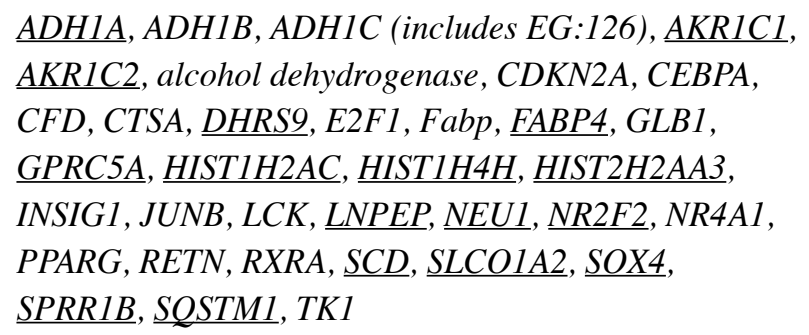 & $\begin{array}{l}\text { Cancer, carbohydrate metabolism, } \\
\text { digestive system development } \\
\text { and function }\end{array}$ & 30 \\
\hline 4 & 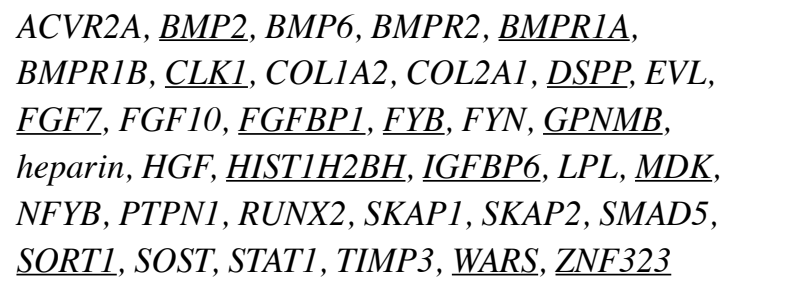 & $\begin{array}{l}\text { Cellular growth and proliferation, } \\
\text { cellular development, } \\
\text { connective tissue development } \\
\text { and function }\end{array}$ & 23 \\
\hline 5 & 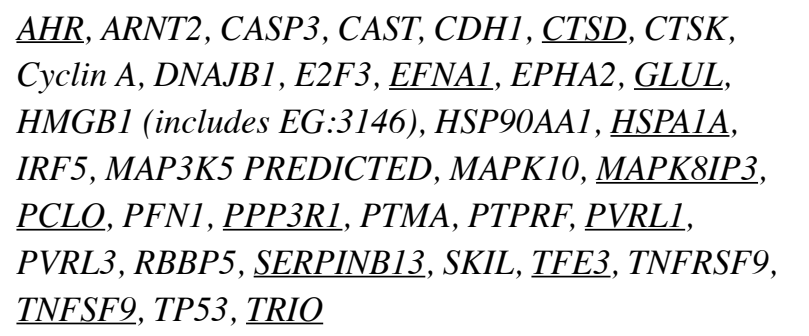 & $\begin{array}{l}\text { Cell death, cellular growth and } \\
\text { proliferation, cellular assembly } \\
\text { and organization }\end{array}$ & 21 \\
\hline 6 & $\begin{array}{l}A R, C a^{2+}, \underline{C A C Y B P}, \underline{C A L B 1}, C D C 37, \text { EXOC1, } \\
\text { EXOC2, EXOC3, EXOC4, EXOC5, EXOC6, EXOC7, } \\
\text { EXOC8, FYN, GSN, Hsp70, Hsp90, HSPA8, MAK, } \\
\text { MBP, NOS3, OASL, PARK2, phosphatidylinositol, } \\
\text { PIK3R1, RALA, S10OA6, S100B, SIAH1, SNCA, } \\
\text { SRC, THRB, TUBA1A, TUBB, VIL2 }\end{array}$ & $\begin{array}{l}\text { Cellular function and maintenance, } \\
\text { cell signaling, molecular transport }\end{array}$ & 9 \\
\hline
\end{tabular}

Underlining indicates genes identified by microarray analysis. ${ }^{a} \mathrm{~A}$ score $>3$ was considered significant.

t-test) after 2, 4, 6 and 8 Gy of irradiation compared with that of corresponding cells treated with siNT (Figs. 4-6). To determine the effect of siLCN2 transfection on cell proliferation, cellular proliferation assay was performed. The data showed no significant effect by siLCN2 transfection on cellular proliferation in Ca9-22 cells (Fig. 7).

\section{Discussion}

Although radiotherapy is considered an effective treatment choice in patients with OSCC, the outcome is not favorable in certain cases. The difference in the outcome mainly depends on the radiosensitivity of tumor cells. Although a set of human genes related to radiosensitivity has been identified (23-29), the 


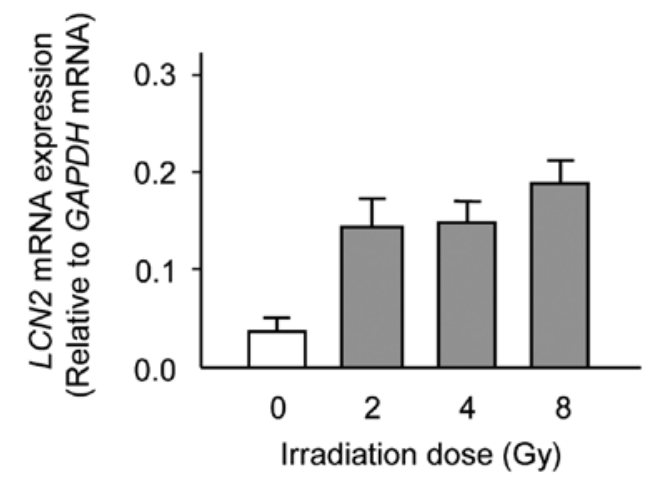

Figure 2. Quantification of $L C N 2 \mathrm{mRNA}$ levels by real-time qRT-PCR analysis of irradiated (2, 4 and $8 \mathrm{~Gy}) \mathrm{Ca} 9-22$ cells. The expression of $L C N 2$ mRNA was upregulated in irradiated Ca9-22 cells as compared to unirradiated Ca9-22 cells.

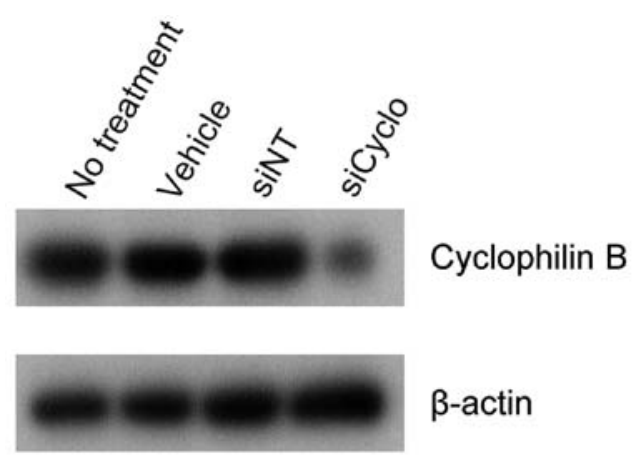

Figure 3. Western blot analysis of cyclophilin B protein in Ca9-22 cells. The cells were transfected with vehicle, siNT, siCyclophilin B (siCyclo) and analyzed after $120 \mathrm{~h}$. The expression of cyclophilin B was diminished in cells transfected with siCyclo. There was no change in the cyclophilin B expression of cells transfected with siNT (negative control siRNA). $\beta$-actin was used as a loading control.

detailed mechanism of radioresistance remains unknown. Thus, the present study aimed to identify molecules that control the response to radiotherapy in OSCC. We identified 167 genes that were upregulated by X-ray irradiation ( $8 \mathrm{~Gy})$ in $\mathrm{Ca} 9-22$ cells by using DNA microarray analysis. We used the IPA tool to analyze the functional networks and gene ontology of these genes, and we detected six genetic networks that were each characterized by different representative functions (Table II). Among the genes, $L C N 2$, which mapped to network 2 , had the greatest increase in expression after X-ray irradiation (Table I). A variety of functions of the LCN2 protein has been reported. These functions include the transport of fatty acids and iron $(30,31)$ and the modulation of inflammatory responses (32). A recent study reported that $L C N 2$ expression was upregulated accompanied with apoptosis induced by several reagents in human adenocarcinoma A549 cells and that the induction of $L C N 2$ represents a survival response (14). Roudkenar et al detected the upregulation of LCN2 expression in $\mathrm{HepG} 2$ cells after the administration of X-rays or $\mathrm{H}_{2} \mathrm{O}_{2}(33)$. These studies suggest that LCN2 defends cells against extracellular stimuli and facilitates cell survival. In the current study, the expression of $L C N 2$ was significantly upregulated by X-ray irradiation (Fig. 2), and $L C N 2$ gene silencing enhanced the radiosensitivity of OSCC cells and lung cancer cells (Figs. 4-6). Thus, LCN2 should also have supported the survival of irradiated cells
A

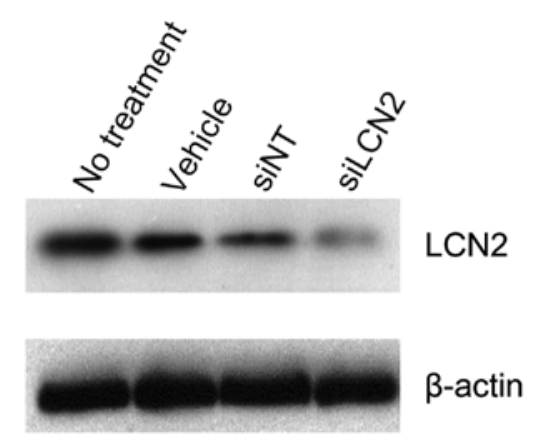

B

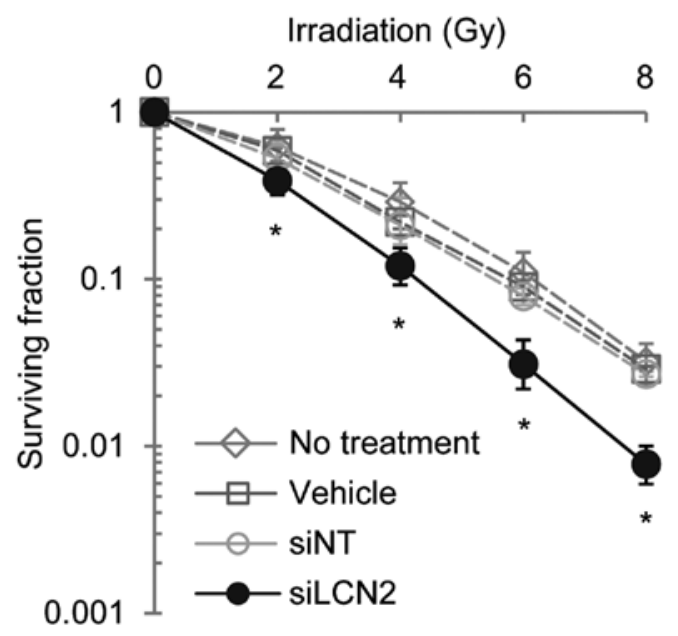

Figure 4. Functional analysis of siLCN2-transfected Ca9-22 cells. (A) Decreased expression of LCN2 protein in siLCN2-transfected Ca9-22 cells was validated by western blot analysis. (B) Survival of siLCN2-transfeced Ca9-22 cells was significantly decreased after 2, 4,6 and 8 Gy of radiation as compared with that of siNT-transfected cells $\left({ }^{*} \mathrm{P}<0.01\right.$, Student's t-test).

in the present study. The biological activity of LCN2 is not cellspecific because the same effect was observed in two different OSCC-derived cell lines and a lung cancer cell line.

Park et al reported that phosphoinositide 3-kinase (PI3K)/Akt mediates the interleukin-3-regulated expression of $24 \mathrm{p} 3$, the mouse analogue of LCN2, in hematopoietic cells (34). Thus, the PI3K/Akt pathway might be closely related to LCN2 expression in solid tumors. We previously reported that the downregulation of ICAM2 expression by siRNA enhanced the radiosensitivity of OSCC cells with an increased apoptotic phenotype via phosphorylation of Akt (13). These studies indicate that the PI3K/ Akt pathway may play a crucial role in the radiosensitivity of OSCC and that LCN2 might be involved in this mechanism.

The current study indicates for the first time that LCN2 is related to radioresistance. Various molecules have been reported to be associated with the radiotherapy response of malignant tumors of the head and neck. p53 DNA contact mutation (10), COX-2 (8), p63 (11) and TCRP1 (12) induced radioresistance, while high survivin expression (9) and downregulated expression of ICAM2 (13) enhanced radiosensitivity. However, 
A
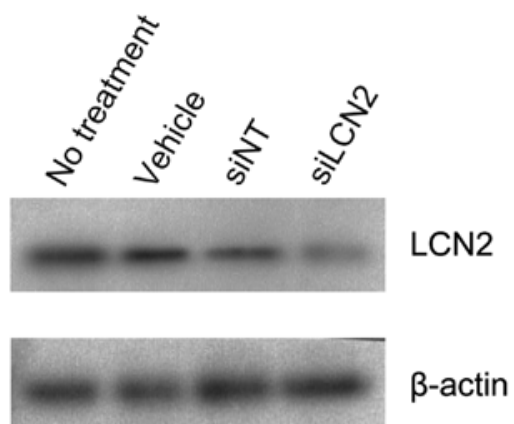

B

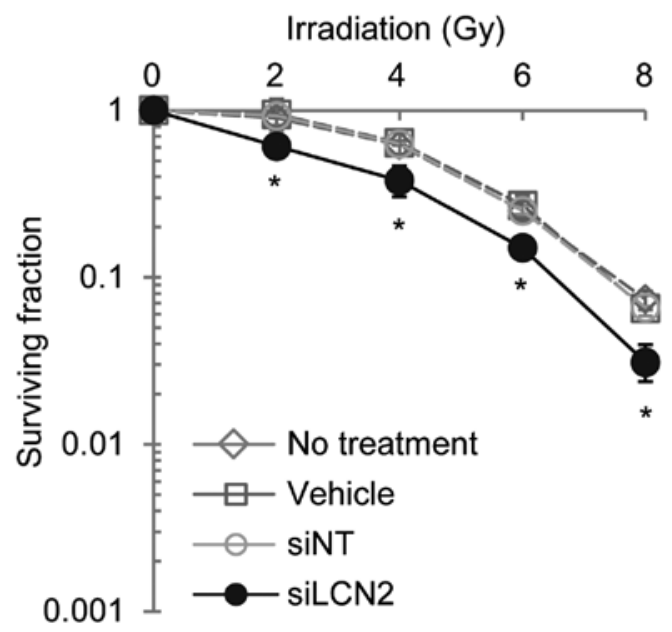

Figure 5. Functional analysis of siLCN2-transfected HSC-2 cells. (A) Decreased expression of LCN2 protein in siLCN2-transfected HSC-2 cells was validated by western blot analysis. (B) Survival of siLCN2-transfeced HSC-2 cells was significantly decreased after 2, 4, 6 and 8 Gy of radiation as compared with that of siNT-transfected cells ( $\mathrm{P}<0.01$, Student's t-test).

the conclusive pathway regulating radioresistance in OSCC has not yet been established, suggesting that the mechanism of responsiveness for irradiation is complex and that various molecules are engaged in the process. Moreover, different subpopulations of tumor cells might have different responses to irradiation. Studies have indicated that cancer stem cells might have key roles in tumor growth, metastasis, progression and chemo-radioresistance (35). Further investigations are needed to clarify subpopulation-dependent characteristics that regulate radioresistance.

In conclusion, we identified genes that are differentially expressed in X-ray irradiated OSCC-derived cell lines. Expression of $L C N 2$ mRNA was significantly greater in irradiated cells than in unirradiated cells, and $L C N 2$ gene silencing enhanced the radiosensitivity of OSCC-derived cell lines and a lung cancer cell line. The current study indicates for the first time that LCN2 is related to radiation response. Our findings suggest that overexpression of LCN2 might contribute to radiation resistance in cancer cells and that $\mathrm{LCN} 2$ could be a diagnostic marker and therapeutic target for OSCC and other
A
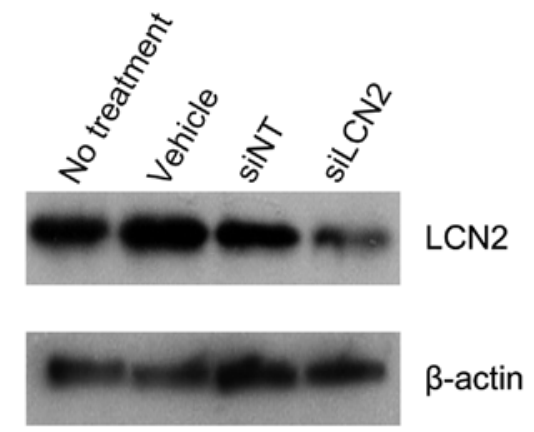

B

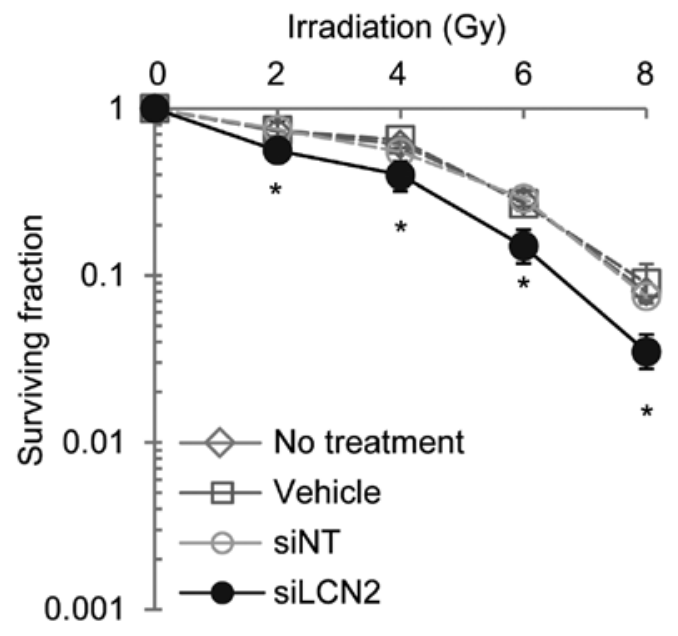

Figure 6. Functional analysis of siLCN2-transfected A549 cells. (A) Decreased expression of LCN2 protein in siLCN2-transfected A549 cells was validated by western blot analysis. (B) Survival of siLCN2-transfected A549 cells was significantly decreased after 2, 4, 6 and 8 Gy of radiation as compared with that of siNT-transfected cells ( $\mathrm{P}<0.01$, Student's t-test).

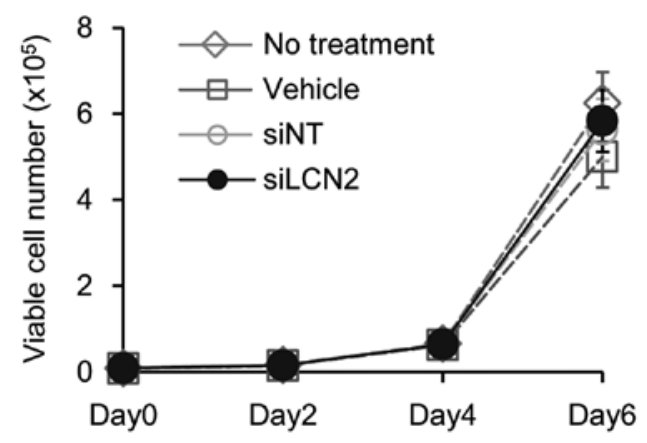

Figure 7. The effect of $L C N 2$ gene silencing on cell proliferation. A cellular proliferation assay was performed to investigate the effect of diminished LCN2 expression on Ca9-22 cells. There was no significant difference in the proliferation rate between siLCN2-transfected cells and control cells.

cancers. This information may lead to the discovery of new target genes and perhaps the development of better radiotherapy strategies for the treatment of cancer. 


\section{References}

1. Lehnert S: Prediction of tumor response to therapy: molecular markers and the microenvironment. Apoptosis and chips: an overview of the proceedings. Radiat Res 154: 121-124, 2000.

2. Joki T, Carroll RS, Dunn IF, Zhang J, Abe T and Black PM: Assessment of alterations in gene expression in recurrent malignant glioma after radiotherapy using complementary deoxyribonucleic acid microarrays. Neurosurgery 48: 195-202, 2001 .

3. Keyse SM: The induction of gene expression in mammalian cells by radiation. Semin Cancer Biol 4: 119-128, 1993.

4. Iliakis G: Cell cycle regulation in irradiated and nonirradiated cells. Semin Oncol 24: 602-615, 1997.

5. Eckardt-Schupp F and Klaus C: Radiation inducible DNA repair processes in eukaryotes. Biochimie 81: 161-171, 1999.

6. Maity A, McKenna WG and Muschel RJ: The molecular basis for cell cycle delays following ionizing radiation: a review. Radiother Oncol 31: 1-13, 1994.

7. Forrester HB, Vidair CA, Albright N, Ling CC and Dewey WC: Using computerized video time lapse for quantifying cell death of X-irradiated rat embryo cells transfected with c-myc or c-Ha-ras. Cancer Res 59: 931-939, 1999.

8. Terakado N, Shintani S, Yano J, et al: Overexpression of cyclooxygenase- 2 is associated with radioresistance in oral squamous cell carcinoma. Oral Oncol 40: 383-389, 2004.

9. Freier K, Pungs S, Sticht C, et al: High survivin expression is associated with favorable outcome in advanced primary oral squamous cell carcinoma after radiation therapy. Int J Cancer 120: 942-946, 2007.

10. Yamazaki Y, Chiba I, Hirai A, et al: Radioresistance in oral squamous cell carcinoma with p53 DNA contact mutation. Am J Clin Oncol 26: e124-e129, 2003.

11. Moergel M, Abt E,Stockinger M and Kunkel M: Overexpression of p63 is associated with radiation resistance and prognosis in oral squamous cell carcinoma. Oral Oncol 46: 667-671, 2010.

12. Gu Y, Fan S, Liu B, et al: TCRP1 promotes radioresistance of oral squamous cell carcinoma cells via Akt signal pathway. Mol Cell Biochem 357: 107-113, 2011.

13. Ishigami T, Uzawa K, Fushimi K, et al: Inhibition of ICAM2 induces radiosensitization in oral squamous cell carcinoma cells. Br J Cancer 98: 1357-1365, 2008.

14. Tong Z, Wu X, Ovcharenko D, Zhu J, Chen CS and Kehrer JP: Neutrophil gelatinase-associated lipocalin as a survival factor. Biochem J 391: 441-448, 2005.

15. Flower DR: The lipocalin protein family: structure and function. Biochem J 318: 1-14, 1996.

16. Triebel S, Blaser J, Reinke H and Tschesche H: A $25 \mathrm{kDa}$ alpha 2 -microglobulin-related protein is a component of the $125 \mathrm{kDa}$ form of human gelatinase. FEBS Lett 314: 386-388, 1992.

17. Nilsen-Hamilton M, Liu Q, Ryon J, Bendickson L, Lepont P and Chang Q: Tissue involution and the acute phase response. Ann NY Acad Sci 995: 94-108, 2003.

18. Nielsen BS, Borregaard N, Bundgaard JR, Timshel S, Sehested $M$ and Kjeldsen L: Induction of NGAL synthesis in epithelial cells of human colorectal neoplasia and inflammatory bowel diseases. Gut 38: 414-420, 1996.

19. Bratt T: Lipocalins and cancer. Biochim Biophys Acta 1482: 318-326, 2000
20. Furutani M, Arii S, Mizumoto M, Kato $\mathbf{M}$ and Imamura $\mathbf{M}$ Identification of a neutrophil gelatinase-associated lipocalin mRNA in human pancreatic cancers using a modified signal sequence trap method. Cancer Lett 122: 209-214, 1998.

21. Bartsch $\mathrm{S}$ and Tschesche $\mathrm{H}$ : Cloning and expression of human neutrophil lipocalin cDNA derived from bone marrow and ovarian cancer cells. FEBS Lett 357: 255-259, 1995.

22. Hiromoto T, Noguchi K, Yamamura M, et al: Upregulation of neutrophil gelatinase-associated lipocalin in oral squamous cell carcinoma: relation to cell differentiation. Oncol Rep 26: 1415-1421, 2011.

23. Ishigami T, Uzawa K, Higo M, et al: Genes and molecular pathways related to radioresistance of oral squamous cell carcinoma cells. Int J Cancer 120: 2262-2270, 2007.

24. Ogawa K, Utsunomiya T, Mimori K, et al: Differential gene expression profiles of radioresistant pancreatic cancer cell lines established by fractionated irradiation. Int J Oncol 28: 705-713, 2006.

25. Guo WF, Lin RX, Huang J, et al: Identification of differentially expressed genes contributing to radioresistance in lung cancer cells using microarray analysis. Radiat Res 164: 27-35, 2005.

26. Hellman B, Brodin D, Andersson M, et al: Radiation-induced DNA-damage and gene expression profiles in human lung cancer cells with different radiosensitivity. Exp Oncol 27: 102-107, 2005.

27. Fukuda K, Sakakura C, Miyagawa K, et al: Differential gene expression profiles of radioresistant oesophageal cancer cell lines established by continuous fractionated irradiation. $\mathrm{Br} \mathrm{J}$ Cancer 91: 1543-1550, 2004

28. Kitahara O, Katagiri T, Tsunoda T, Harima Y and Nakamura Y: Classification of sensitivity or resistance of cervical cancers to ionizing radiation according to expression profiles of 62 genes selected by cDNA microarray analysis. Neoplasia 4: 295-303, 2002.

29. Achary MP, Jaggernauth W, Gross E, Alfieri A, Klinger HP and Vikram B: Cell lines from the same cervical carcinoma but with different radiosensitivities exhibit different cDNA microarray patterns of gene expression. Cytogenet Cell Genet 91: 39-43, 2000

30. Chu ST, Lin HJ, Huang HL and Chen YH: The hydrophobic pocket of $24 \mathrm{p} 3$ protein from mouse uterine luminal fluid: fatty acid and retinol binding activity and predicted structural similarity to lipocalins. J Pept Res 52: 390-397, 1998.

31. Yang J, Goetz D, Li JY, et al: An iron delivery pathway mediated by a lipocalin. Mol Cell 10: 1045-1056, 2002.

32. Cowland JB and Borregaard N: Molecular characterization and pattern of tissue expression of the gene for neutrophil gelatinase-associated lipocalin from humans. Genomics 45: 17-23, 1997.

33. Roudkenar MH, Kuwahara Y, Baba T, et al: Oxidative stress induced lipocalin 2 gene expression: addressing its expression under the harmful conditions. J Radiat Res 48: 39-44, 2007.

34. Park S, Guo J, Kim D and Cheng JQ: Identification of 24p3 as a direct target of Foxo3a regulated by interleukin-3 through the phosphoinositide 3-kinase/Akt pathway. J Biol Chem 284: 2187-2193, 2009

35. Rosen JM and Jordan CT: The increasing complexity of the cancer stem cell paradigm. Science 324: 1670-1673, 2009. 